\title{
Tracing the evolution of tissue identity with microRNAs
}

\author{
Katrien De Mulder and Eugene Berezikov*
}

\section{Abstract \\ Comparison of microRNA expression identified tissues present in the last common ancestor of Bilaterians and put evolution of microRNAs in the context of tissue evolution.}

Animal evolution has fascinated biologists for centuries and, despite tremendous progress in our understanding of the evolutionary process, it still keeps many of its mysteries secret. Initially, morphological and developmental studies were performed to reconstruct the road that animal evolution has followed. With the coming of age of molecular biology, comparative single- and multiplegene analyses contributed to the further unraveling of evolutionary relationships within the animal kingdom. Although these studies resulted in the separation of the main phyla and taxa, the occurrence of convergent evolution, secondary loss of characters, poor knowledge of several animal groups at key positions and the presence of slow- and fast-evolving genomes complicated the reconstruction of the exact evolutionary paths.

Over the past decade, it has become clear that the appearance of more complex organisms during animal evolution was driven by an increase in the complexity of gene regulatory mechanisms [1] at both a transcriptional and a post-transcriptional level [2]. Intriguingly, mechanisms of post-transcriptional gene regulation by noncoding RNAs were already present early on in the evolution of the Metazoa [3]. In particular, microRNAs (miRNAs) have been suggested to have a major role in evolutionary changes of body structure, as the number of miRNA genes correlates strikingly with the morphological complexity of organisms [4-6]. miRNAs are small 21 to 23 nucleotide non-coding RNAs that regulate gene expression by binding to specific target mRNAs, leading

*Correspondence: e.berezikov@hubrecht.eu

Hubrecht Institute and University Medical Center Utrecht, Uppsalalaan 8, 3584CT Utrecht, The Netherlands to their translational inhibition and/or degradation. Given that miRNAs control gene expression in a wide range of biological processes, including developmental timing, cell proliferation and differentiation, it is feasible that alterations in spatio-temporal expression of miRNAs during evolution could result in significant changes in physiology and morphology between different taxa.

Novel miRNAs continuously evolve in animal genomes [7]. Once integrated into a gene regulatory network, miRNAs are strongly conserved and not susceptible to significant secondary loss. As such, miRNA studies partially overcome the limitations faced by morphological, developmental and protein comparison approaches, such as parallel evolution, convergence and missing data. These appealing characters rapidly attracted the attention of evolutionary biologists, and miRNAs became a promising tool for reconstructing animal evolution.

\section{The coming age of miRNAs in evolutionary studies}

In a recent study, Christodoulou et al. [8] have begun to assess the correlation between expression patterns of ancient miRNAs and body-plan evolution in Bilateria. The Bilateria mainly consists of protostomes and deuterostomes, which are collectively called nephrozoans, plus a few basal phyla, such as acoels, nemertodermatids and chaetognaths (Figure 1). In their comparative approach, Christodoulou et al. [8] focused on miRNAs conserved between the two major superphyla within the Bilateria protostomes (for example, arthropods, nematodes and molluscs) and deuterostomes (for example, vertebrates and echinoderms). The authors hypothesized that any specific localization shared between protostomes and deuterostomes should reflect an ancient specificity of that miRNA in their last common ancestor. To address this question, they used the annelids Platynereis dumerilii and Capitella sp. (new representatives of the understudied lophotrochozoan protostomes) and the sea urchin Strongylocentrotus purpuratus (basal representative of the deuterostomes), with the cnidarian Nematostella vectensis as an outgroup for the Bilateria.

Initially, the authors [8] performed deep sequencing of the small RNA repertoire to identify the conserved 


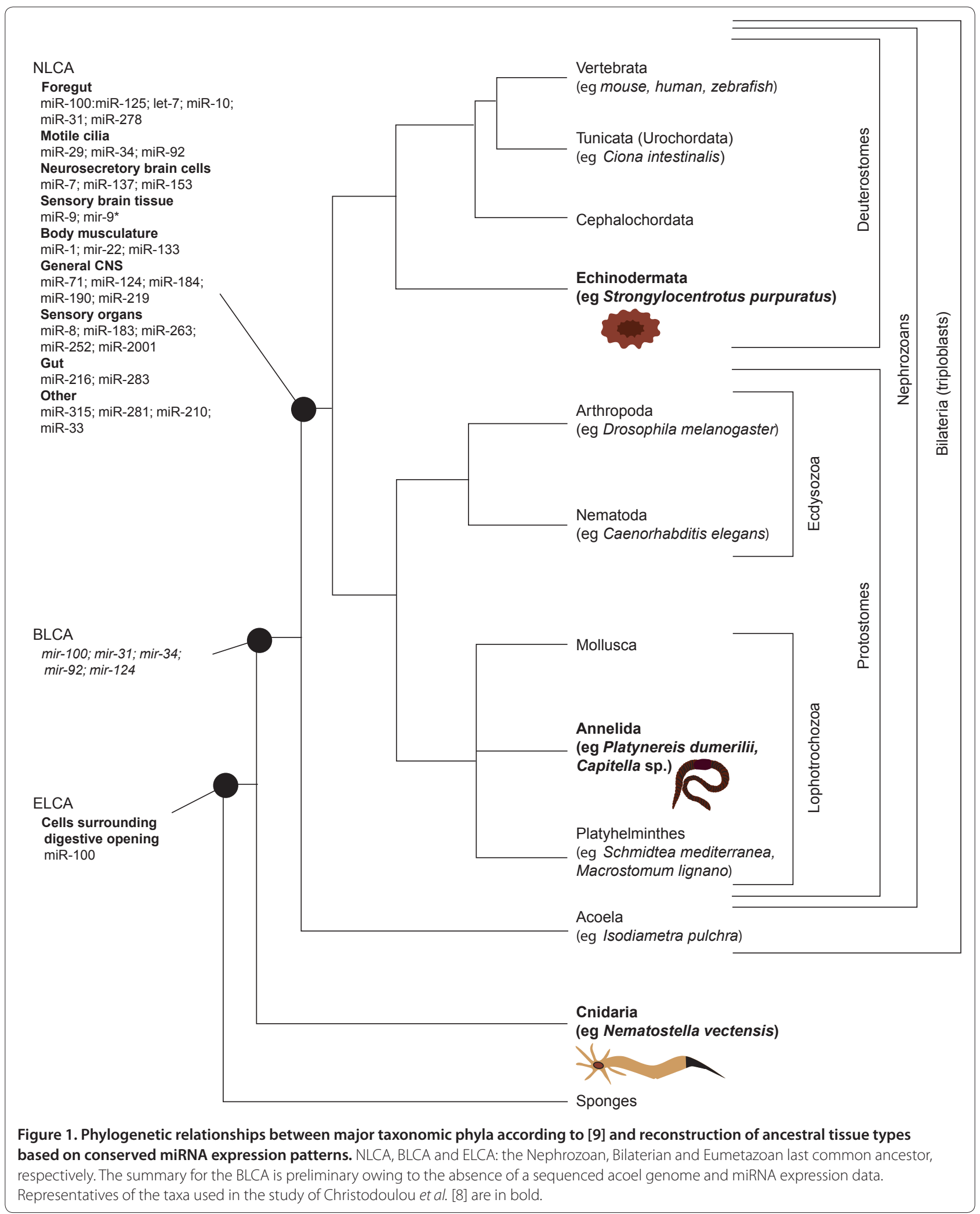

bilaterian miRNAs, and found, in accordance with recent studies [3-6], 34 miRNA families common to protostomes and deuterostomes. Subsequently, they investigated in detail the spatio-temporal localization profile of these 
conserved miRNAs in Platynereis using whole mount in situ hybridization and found that expression patterns of these miRNAs are highly specific for certain tissues and cell types and are strongly conserved throughout bilaterian evolution.

This comparison allowed Christodoulou and colleagues [8] to reconstruct the minimal set of cell types and tissues that existed in the last common ancestor of nephrozoans (Figure 1). This ancestor is predicted to have had neurosecretory cells along its mouth (characterized by the expression of miR-100, miR-125 and let-7) and motile ciliated cells (miR-29+ miR-34+ miR-92+). In addition, the nephrozoan ancestor would have had a miR-1 $1^{+}$miR-22+ miR- $133^{+}$body musculature, a miR- $12^{+}$miR- $216^{+}$ miR- $283^{+}$gut and miR- $9^{+}$miR- $9^{*+}$ cells related to sensory information processing. Finally, the nephrozoan ancestor is predicted to have had a miR-124+ central nervous system, which would be connected with a miR- $8^{+}$ miR- $183^{+}$miR- $263^{+}$peripheral sensory tissue, and to be already equipped with neurosecretory cells in a primitive brain (miR- $7^{+}$miR-137 ${ }^{+}$miR-153+).

\section{Implications and new directions}

Innovation at the post-transcriptional gene regulatory level through expansion of the miRNA repertoire has previously been suggested as one of the driving forces behind the evolution of animal complexity [3-7]. It is not clear, however, how exactly novel miRNAs evolve and what roles they have in the establishment of tissue identity. According to the model of transcriptional control of new miRNA genes suggested by Chen and Rajewsky [2], newly emerging miRNAs initially should be expressed at low levels and in specific tissues in order to minimize deleterious off-targeting effects and to allow natural selection to eliminate these slightly deleterious targets over time. Subsequently, miRNA expression levels can be increased and tissue-specificity relaxed [2]. Now, with the discovery of Christodoulou et al. [8] that ancient miRNAs were expressed in specific cell types of the protostomedeuterostome ancestor and in many cases assumed broader expression patterns later in evolution, this model of miRNA emergence gains additional solid experimental support.

As shown by Christodoulou et al. [8], comparison of the miRNA repertoire between different taxa can significantly contribute to the hypothetical reconstruction of the ancestral body plan: by a detailed examination in which tissues/cell types conserved miRNAs evolved, the authors [8] were able to create a hypothetical picture of an ancestor at a key phylogenetic position for which we have no fossils. Although the appearance of the last common ancestor of deuterostomes and protostomes still remains elusive, the authors [8] elucidated the differentiated cell repertoire from this ancestor and, by doing so, unequivocally established miRNAs as a powerful new tool for reconstructing ancient animal body plans at important evolutionary nodes. Further investigation of miRNA repertoires and expression patterns in additional taxa might give fundamental clues about unknown nodes within the animal tree and resolve some phylogenetic uncertainties.

For example, one of the frequently disputed questions is the phylogenetic position of Acoelomorpha (which includes the flatworm-like acoels and nemertodermatids). Acoels were originally grouped within the phylum Platyhelminthes but have recently been placed at a key position at the base of the Bilateria on the basis of new molecular data [9] (Figure 1). Earlier studies revealed that the highly conserved miRNA let-7, which is present in all other Bilaterians, is absent in acoels, indicating that acoels might have branched off earlier from the last common ancestor of protostomes and deuterostomes. In addition, although acoels are believed to primitively lack a real brain, having instead a simple 'commissural' brain characterized by transverse fiber accumulation in the head, without classical ganglionic cell mass [10], Christodoulou et al. [8] suggest that nervous system centralization was already present before the split between protostomes and deuterostomes. Therefore, a detailed analysis of the acoel miRNA repertoire and their corresponding expression patterns might help to further reveal how evolution at the base of the Bilateria took place and whether or not the urbilaterian - the last common ancestor of acoels and nephrozoans - had complex tissues.

Conservation of sequence and expression patterns suggests that the core functions of ancient miRNAs also remained conserved through evolution. What are these core functions? From data from other animal models, Christodoulou et al. [8] speculate that some miRNAs, such as miR-100 and let-7, could have roles in developmental timing. However, only few miRNA genes are known to work as developmental switches, and, perhaps surprisingly, the majority of miRNAs are in fact not essential for initial establishment of tissue identity but seem to be important for the maintenance of cells in differentiated states. It is likely, then, that miRNAs facilitate evolution of complexity by stabilizing existing and newly emerging regulatory circuits and transcriptional programs. Elucidating the principle components of miRNA-containing networks that were present at the dawn of animal evolution and tracing the acquisition of new miRNA circuitry through evolution is the next great evo-devo challenge in the miRNA field.

Acknowledgments

We thank Bernhard Egger and Turan Demircan for fruitful discussions.

Published: 30 March 2010 


\section{References}

1. Levine M, Tjian R: Transcription regulation and animal diversity. Nature 2003, 424:147-151

2. Chen K, Rajewsky N: The evolution of gene regulation by transcription factors and microRNAs. Nat Rev Genet 2007, 8:93-103.

3. Grimson A, Srivastava M, Fahey B, Woodcroft BJ, Chiang HR, King N, Degnan BM, Rokhsar DS, Bartel DP: Early origins and evolution of microRNAs and Piwi-interacting RNAs in animals. Nature 2008, 455:1 193-1197.

4. Sempere LF, Cole CN, McPeek MA, Peterson KJ: The phylogenetic distribution of metazoan microRNAs: insights into evolutionary complexity and constraint. J Exp Zool B Mol Dev Evol 2006, 306:575-588.

5. Prochnik SE, Rokhsar DS, Aboobaker AA: Evidence for a microRNA expansion in the bilaterian ancestor. Dev Genes Evol 2007, 217:73-77.

6. Heimberg AM, Sempere LF, Moy VN, Donoghue PC, Peterson KJ: MicroRNAs and the advent of vertebrate morphological complexity. Proc Natl Acad SC USA 2008, 105:2946-2950.

7. Berezikov E, Thuemmler F, van Laake LW, Kondova I, Bontrop R, Cuppen E, Plasterk RH: Diversity of microRNAs in human and chimpanzee brain. Nat
Genet 2006, 38:1375-1377.

8. Christodoulou F, Raible F, Tomer R, Simakov O, Trachana K, Klaus S, Snyman H, Hannon GJ, Bork P, Arendt D: Ancient animal microRNAs and the evolution of tissue identity. Nature 2010, 463:1084-1048.

9. Egger B, Steinke D, Tarui H, De Mulder K, Arendt D, Borgonie G, Funayama N, Gschwentner R, Hartenstein V, Hobmayer B, Hooge M, Hrouda M, Ishida S, Kobayashi C, Kuales G, Nishimura O, Pfister D, Rieger R, Salvenmoser W, Smith J, Technau U, Tyler S, Agata K, Salzburger W, Ladurner P: To be or not to be a flatworm: the acoel controversy. PLoS ONE 2009, 4:e5502.

10. Raikova OI, Reuter M, Kotikova EA, Gustafsson MKS: A commissural brain! The pattern of 5-HT immunoreactivity in Acoela (Plathelminthes). Zoomorphology 1998, 118:69-77.

doi:10.1186/gb-2010-11-3-111

Cite this article as: De Mulder K, Berezikov E: Tracing the evolution of tissue identity with microRNAs. Genome Biology 2010, 11:111. 